\title{
Prone position ameliorates lung elastance and increases functional residual capacity independently from lung recruitment
}

\author{
Alessandro Santini ${ }^{1 *}$, Alessandro Protti ${ }^{2}$, Thomas Langer ${ }^{2}$, Beatrice Comini ${ }^{1}$, Massimo Monti ${ }^{1}$, Cristina Carin Sparacino ${ }^{1}$, \\ Daniele Dondossola ${ }^{3}$ and Luciano Gattinoni ${ }^{1,2}$
}

\footnotetext{
* Correspondence:

alessandro.santini@unimi.it

'Dipartimento di Fisiopatologica

Medico-Chirurgica e dei Trapianti,

Università degli Studi di Milano, Via

Francesco Sforza 35, 20122 Milan,

Italy

Full list of author information is

available at the end of the article
}

\begin{abstract}
Background: Prone position is used to recruit collapsed dependent lung regions during severe acute respiratory distress syndrome, improving lung elastance and lung gas content. We hypothesised that, in the absence of recruitment, prone position would not result in any improvement in lung mechanical properties or gas content compared to supine position.

Methods: Ten healthy pigs under general anaesthesia and paralysis underwent a pressure-volume curve of the respiratory system, chest wall and lung in supine and prone positions; the respective elastances were measured. A lung computed tomography (CT) scan was performed in the two positions to compute gas content (i.e. functional residual capacity $(F R C)$ ) and the distribution of aeration. Recruitment was defined as a percentage change in non-aerated lung tissue compared to the total lung weight.

Results: Non-aerated (recruitable) lung tissue was a small percentage of the total lung tissue weight in both positions ( $4 \pm 3$ vs $1 \pm 1 \%$, supine vs prone, $p=0.004$ ). Lung elastance decreased ( $20.5 \pm 1.8$ vs $15.5 \pm 1.6 \mathrm{cmH}_{2} \mathrm{O} / \mathrm{l}$, supine vs prone, $\left.p<0.001\right)$ and functional residual capacity increased (380 \pm 82 vs $459 \pm 60 \mathrm{ml}$, supine vs prone, $p=0.025)$ in prone position; specific lung elastance did not change (7.0 \pm 0.5 vs $6.5 \pm$ $0.5 \mathrm{cmH}_{2} \mathrm{O}$, supine vs prone, $\left.p=0.24\right)$. Lung recruitment was low ( $\left.3 \pm 2 \%\right)$ and was not correlated to increases in functional residual capacity $\left(R^{2} 0.2, p=0.19\right)$. A higher amount of well-aerated and a lower amount of poorly aerated lung tissue were found in prone position.
\end{abstract}

Conclusions: In healthy pigs, prone position ameliorates lung mechanical properties and increases functional residual capacity independently from lung recruitment, through a redistribution of lung aeration.

Keywords: Prone position; Supine position; Lung recruitment; Pressure-volume curve; Lung elastance; Functional residual capacity

\section{Background}

Prone position is used as a rescue therapy during acute respiratory distress syndrome (ARDS) in severely hypoxic patients $[1,2]$ in whom it usually improves oxygenation and lung mechanics $[3,4]$. The oxygenation benefit is due to a better ventilation-perfusion matching $[5,6]$ and/or to recruitment of dorsal lung parenchyma with a decrease in shunt fraction $[4,7,8]$. The change in the mechanical properties of the lung is usually

\section{Springer}

(c) 2015 Santini et al. This is an Open Access article distributed under the terms of the Creative Commons Attribution License (http:// creativecommons.org/licenses/by/4.0), which permits unrestricted use, distribution, and reproduction in any medium, provided the original work is properly credited. 
attributed to lung recruitment with an increase in lung resting volume $[3,9]$ and to a lower vertical pleural pressure gradient in prone position, with consequent more homogenous distribution of transpulmonary pressure, lung inflation and thus ventilation $[5,6,10]$.

Since the first description of ARDS, it became evident that mechanical ventilation per se can worsen lung damage, spread systemic inflammation and affect outcome [1] and a new nosologic entity was defined, namely ventilator-induced lung injury (VILI). Many animal studies either on healthy or diseased lungs have reported the role of prone position in delaying VILI appearance [11, 12] or in reducing its severity [13-15].

The determinants of VILI are excessive pressures acting on lung parenchyma (i.e. transpulmonary pressure or stress) and lung deformation over lung resting volume (i.e. tidal volume/functional residual capacity or strain), closely linked to each other by lung intrinsic mechanical properties (i.e. specific lung elastance) [16, 17]. The protective effect of prone position could accordingly be due either to an increase in lung resting volume (i.e. functional residual capacity (FRC)) with a consequent decrease in strain and transpulmonary pressure for the same tidal volume applied or to the prevention of opening and closing of lung units during tidal ventilation (atelectrauma).

Both mechanisms of protection (increase in FRC with decrease in transpulmonary pressure and reduction of atelectrauma) are usually thought to depend on the presence of recruitable lung tissue, i.e. non-aerated, collapsed lung units which undergo reopening and aeration during the respiratory cycle [18], and should have, if any, a marginal role in healthy lungs or in diseased lungs with low recruitable tissue.

In this study, we investigated lung mechanical properties and volumes in supine and prone positions in a healthy animal model, with a small amount of non-aerated, potentially recruitable lung tissue. Our hypothesis was that, in the absence of potential for lung recruitment, prone position would not result in any change in lung mechanical behaviour and lung resting volume compared to supine position. In contrast with our hypothesis, we found that even when recruitment is negligible, prone position ameliorates lung elastance and increases functional residual capacity, mainly through a different, more homogenous distribution of lung aeration.

\section{Methods}

The study was approved by the Institutional Review Board and was conducted in accordance to international recommendations on animal care [19].

Ten healthy piglets weighing $21 \pm 2 \mathrm{~kg}$ were studied. Anaesthesia induction and surgical preparation were performed as previously described, in supine position [16]. A 5-cm-long latex oesophageal balloon was placed in the lower third of the oesophagus to estimate changes in pleural pressure. Proper positioning was confirmed with computed tomography (CT) scan (see below).

Each animal was studied both in supine and prone positions, after a recruitment manoeuvre ( $1 \mathrm{~min}$ of pressure-controlled ventilation with inspiratory pressure $40 \mathrm{cmH}_{2} \mathrm{O}$, PEEP $5 \mathrm{cmH}_{2} \mathrm{O}, \mathrm{I}: \mathrm{E}=0.5, \mathrm{~F}_{\mathrm{i}} \mathrm{O}_{2}=0.5$ ). A static pressure (airway and oesophageal)-volume curve was recorded with a dedicated software (Colligo, www.elekton.it). A 100-ml calibrated glass syringe was attached to the endotracheal tube, and 100-ml aliquots of room air were inflated into the lungs until an airway pressure of $35 \mathrm{cmH}_{2} \mathrm{O}$ was reached. Each step lasted $5 \mathrm{~s}$, reaching static conditions. 
From airway $\left(P_{\mathrm{aw}}\right)$ and oesophageal $\left(P_{\mathrm{es}}\right)$ pressure recordings, transpulmonary pressure $\left(P_{\mathrm{L}}\right)$ was calculated as:

$$
P_{\mathrm{L}}=\left(P_{\mathrm{aw}}-P_{\mathrm{aw}, \mathrm{ZEEP}}\right)-\left(P_{\mathrm{es}, \mathrm{vol}}-P_{\mathrm{es}, \mathrm{ZEEP}}\right)
$$

where $P_{\text {aw }}$ is the airway pressure, $P_{\text {aw,ZEEP }}$ is the airway pressure at end expiration (verified to be always equal to the atmospheric pressure), $P_{\mathrm{es}, \mathrm{vol}}$ is the oesophageal pressure at any given inflation volume and $P_{\text {es,ZEEP }}$ is the oesophageal pressure at end expiration.

For each pig, the respiratory system $\left(P_{\mathrm{aw}}-\right.$ volume), chest wall ( $P_{\mathrm{es}}-$ volume) and lung $\left(P_{\mathrm{L}}\right.$-volume) pressure-volume curves in prone and supine positions were computed. The slope of the linear part (by visual inspection) of each pressure-volume curve was used to measure the respective elastance value. Once functional residual capacity was measured (see below), specific lung elastance was computed as the slope of the linear part (by visual inspection) of the stress-strain curve.

After an additional recruitment manoeuvre, a lung CT scan was performed at end expiration with the endotracheal tube clamped, in prone and supine positions. CT scan settings have been described elsewhere [16, 20], and lung CT scan-derived lung weight measurement has been previously validated in this same animal model by our group [21].

Lung CT images were manually outlined including only lung parenchyma and excluding bronchi and big intrapulmonary vessels and analysed with a dedicated software (Maluna 3.17, University Hospital of Göttingen, Germany) to measure lung gas content (i.e. functional residual capacity), lung weight and aeration distribution, as previously described [22]. Lung tissue was divided into four compartments based on the degree of aeration: non-aerated tissue (density from 0 to -100 H.U.), poorly aerated tissue (density from -101 to -500 H.U.), well-aerated tissue (density from -501 to -900 H.U.) and overaerated tissue (density from -901 to -1000 H.U.). Lung tissue weight of each compartment was expressed as a percentage of the total lung weight. We defined recruitment as:

Lung recruitment $(\%)=$ (non-aerated lung tissue weight $(\mathrm{g})$ in supine position - non-aerated lung tissue weight $(\mathrm{g})$ in prone position)

/total lung weight $(\mathrm{g})$ in supine position $\times 100$

The coefficient of variation of voxel density distribution, used as a surrogate measure of homogeneity of aeration distribution, was calculated as the standard deviation of CT densities divided by mean CT density in each position.

\section{Statistical analysis}

Data are reported as mean \pm standard deviation unless otherwise stated. Variables acquired in the two positions were compared with paired Student's $t$ test. The change in weight of each lung compartment (non-aerated, poorly aerated, well-aerated and over-aerated) between the two positions is always expressed as a percentage change compared to the total lung weight. Linear regression was used to correlate changes in normally distributed variables between the two positions.

Statistical analysis was performed with Stata software (StataCorp. 2013. Stata Statistical Software: Release 13. College Station, TX: StataCorp LP.). 


\section{Results}

Ten healthy pigs were studied in supine and prone positions. Non-aerated, potentially recruitable lung tissue weight was less than $5 \%$ of the total lung weight in both positions, and lung recruitment was negligible ( $3 \pm 2 \%$ passing from supine to prone position).

Mean respiratory system elastance was not different in the two positions. However, chest wall elastance worsened $\left(10.6 \pm 1.0\right.$ vs $17.0 \pm 0.7 \mathrm{cmH}_{2} \mathrm{O} / \mathrm{l}$, supine vs prone, $p<0.001)$, and lung elastance ameliorated $\left(20.5 \pm 1.8\right.$ vs $15.5 \pm 1.6 \mathrm{cmH}_{2} \mathrm{O} / \mathrm{l}$, supine vs prone, $p<0.001$ ) in prone position (Fig. 1).

A higher gas content, i.e. functional residual capacity, was found ( $380 \pm 82$ vs $459 \pm$ $60 \mathrm{ml}$, supine vs prone, $p=0.025$ ), in spite of a similar amount of aerated lung tissue, due to a different distribution of gas in the alveoli in prone compared to supine position (Table 1). A more homogeneous distribution of aeration in prone position is indicated by the lower coefficient of variation of voxel density distribution (Fig. 2).

The change in non-aerated lung tissue weight, i.e. lung recruitment, passing from supine to prone position did not correlate with the observed increase in FRC $\left(R^{2} 0.2\right.$, $p=0.19)$. The change in poorly aerated lung tissue weight, instead, correlated inversely with FRC change $\left(R^{2} 0.41, p=0.046\right)$ while the change in well-aerated lung tissue weight correlated directly with FRC change $\left(R^{2} 0.53, p=0.02\right)$.

Specific (intrinsic) lung elastance was not different $\left(7.0 \pm 0.5\right.$ vs $6.5 \pm 0.5 \mathrm{cmH}_{2} \mathrm{O}$, supine vs prone, $p=0.24$ ), as shown by the similar slope of the mean stress-strain curve in the two positions (Fig. 3).

\section{Discussion}

In this healthy animal model, the changes in lung mechanics, lung volume and distribution of aeration exclusively due to a change in body position-prone vs supine-were studied. In contrast with our hypothesis, we found a change in lung elastance and functional residual capacity even in the absence of lung recruitment.

Lung mechanical properties improved in prone position, as shown by the different slopes of the lung pressure-volume curve, corresponding to a decrease in lung elastance.

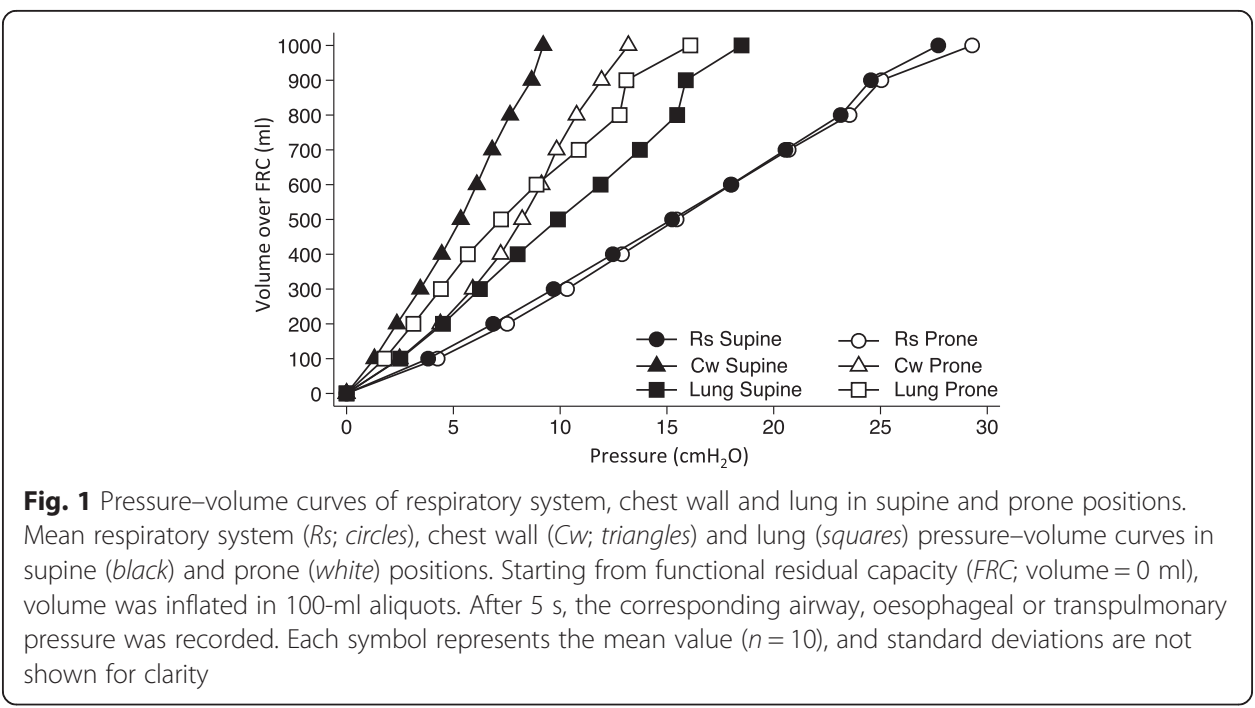


Table 1 Lung volume, weight and distribution of aeration in supine and prone positions

\begin{tabular}{llll}
\hline & Supine & Prone & $p$ value \\
\hline Total lung weight (g) & $345 \pm 26$ & $358 \pm 44$ & 0.420 \\
Aerated lung weight (g) & $330 \pm 24$ & $353 \pm 43$ & 0.156 \\
Functional residual capacity (ml) & $380 \pm 82$ & $459 \pm 60$ & 0.025 \\
Non-aerated lung tissue (\% of total) & $4 \pm 3$ & $1 \pm 1$ & 0.004 \\
Poorly aerated lung tissue (\% of total) & $47 \pm 13$ & $30 \pm 9$ & 0.005 \\
Well-aerated lung tissue (\% of total) & $49 \pm 15$ & $68 \pm 10$ & 0.003 \\
Over-aerated lung tissue (\% of total) & $0 \pm 0$ & $0 \pm 0$ & 0.432 \\
\hline
\end{tabular}

Lung CT scan-derived values: lung volume, lung tissue weight and lung tissue compartments in prone and supine positions. Aerated lung weight was defined as lung weight having a density $<-100 \mathrm{H}$.U. Lung tissue compartments were divided based on their densities (see text), and the weight of each compartment was expressed as a percentage of the total lung weight. The difference between non-aerated lung tissue weights in the two positions expressed as a percentage of the total lung weight corresponds to lung recruitment (see text)

An expected worsening in chest wall mechanical properties, i.e. an increase in chest wall elastance, of approximately the same magnitude, paralleled this effect. These two opposite behaviours summed to give a net negligible result on respiratory system elastance. Thus, if partitioning of respiratory system mechanics in lung and chest wall components had not been performed, it would have been impossible to appreciate any change between supine and prone positions (Fig. 1).

This unexpected change in lung mechanical properties in a healthy animal model led us to consider the possibility that functional residual capacity had increased in prone position and to look for lung recruitment as a possible explanation for this increase in lung gas content. Lung recruitment however depends on the amount of non-aerated lung tissue (i.e. on the number of potentially recruitable lung units) [23], which should be low to nil in healthy lungs. In order to clarify whether lung recruitment had occurred, lung CT scans were performed and lung weight, gas content and distribution of aeration were measured.

Functional residual capacity was actually higher in prone than in supine position. The amount of non-aerated lung tissue was a small percentage of the total lung tissue

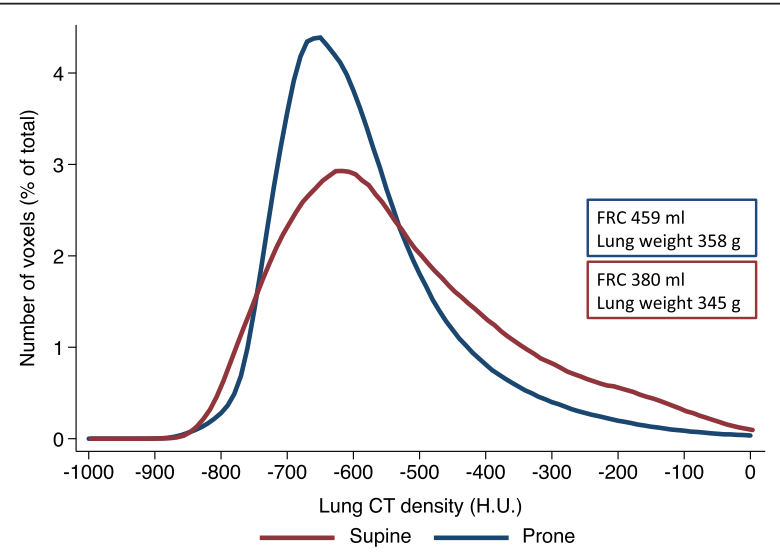

Fig. 2 Frequency distribution of voxels in supine and prone positions. Mean number of voxels, expressed as a percentage of the total number of voxels, for every 10 H.U. changes in CT-derived lung density, in supine (red) and prone (blue) positions. The red and blue inserts refer to the mean values of $F R C$ and lung weight in supine and prone positions, respectively. The coefficient of variation of voxel distribution was 0.32 in supine and 0.23 in prone positions 


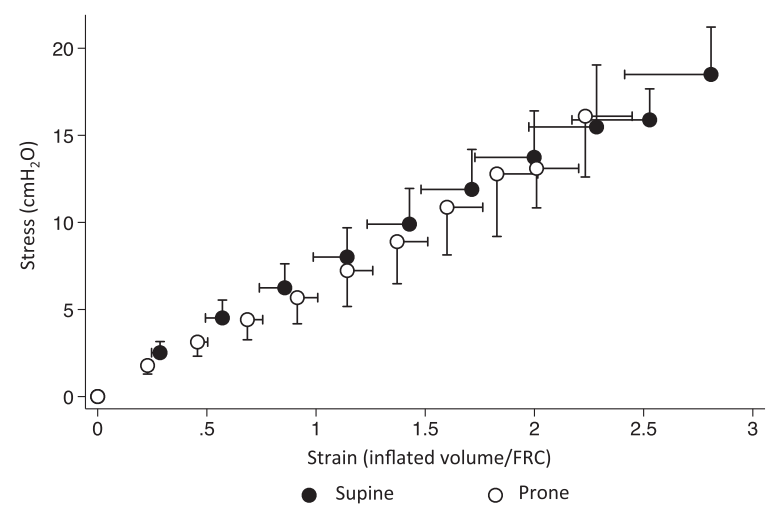

Fig. 3 Stress-strain curve in supine and prone positions. Mean and standard error bars $(n=10)$ of the stress-strain curve in supine (black) and prone (white) positions. Strain was calculated as volume over FRC/ FRC; stress was equal to transpulmonary pressure $\left[P_{\mathrm{L}}=\left(P_{\text {aw }}-P_{\text {aw, ZEEP }}\right)-\left(P_{\text {es,vol }}-P_{\text {es, ZEEP }}\right)\right]$ (see text). Each dot represents a 100-ml step of lung inflation; pressure was recorded after static conditions were reached. The slope of the linear part of the stress-strain curve, which corresponds to specific lung elastance, is not different between prone and supine positions

weight, as expected in healthy lungs, and even if changing position from supine to prone resulted in modest lung recruitment, this did not correlate with functional residual capacity change. Altogether, these results make very unlikely that the opening of previously collapsed lung units, corresponding to lung CT scan analysis of non-aerated lung tissue ( $\geq-100$ H.U.) which becomes aerated (<-100 H.U.), substantially accounted for the observed increase in FRC. Instead, the opposite changes in poorly and wellaerated lung tissue showed a significant correlation with functional residual capacity change. As the total mass of aerated lung tissue did not change, these density modifications anatomically correspond to already open lung units that get more inflated in prone position (poorly aerated lung tissue which becomes well aerated). This might be considered as a different kind of "recruitment", which we could define as a redistribution of aeration, i.e. a large amount of lung tissue with poor aeration in supine position, which becomes well aerated in prone position. As with recruitment, when redistribution of aeration occurs, lung gas content increases, but differently from recruitment, the total amount of lung tissue open to aeration does not change. The differences between these two phenomena merit some further explanation: when previously collapsed lung units reopen, the same amount of gas distributes to a higher number of lung units (a larger lung open to aeration), giving as net effect a decrease in pressure. This would typically be seen on the pressure-volume curve as a flat step (an increase in volume without an increase in pressure), which clearly did not happen in our animals, since the lung pressure-volume curves in supine and prone positions never get close to each other.

The mechanical advantage given by the redistribution of aeration instead is probably related to other phenomena, since the total number of lung units open to aeration (i.e. the dimensions of the lung open to aeration) does not change. These might be an increase in the radius of the alveoli open to aeration, which lowers the elastance of each alveolus, and a higher homogeneity in the distribution of aeration (see Fig. 2), which through interdependence affects the elastance of higher radius alveoli close to lower radius alveoli.

We do not know whether the major contributor to lung mechanical improvement is the increase in volume itself, or the "quality" of the lung tissue open to aeration, since well- 
aerated lung tissue, not poorly aerated, has been associated to the mechanical properties of the lung in patients with ARDS [22]. We however propose redistribution of aeration as a mechanism, different from classically defined lung recruitment, which could explain an amelioration of lung elastance and an increase in lung gas content associated with prone positioning, even in those situations in which a very low amount of non-aerated recruitable lung tissue is present and recruitment is expected to be low.

\section{Conclusions}

In this healthy animal model in which lung recruitment was negligible, prone position was associated with an amelioration of lung mechanics, a higher functional residual capacity and a different, more homogenous distribution of lung aeration than supine position. Lung recruitment, defined as an opening of previously collapsed lung units, is thus not necessary per se for prone position to improve lung mechanical properties and increase lung gas content.

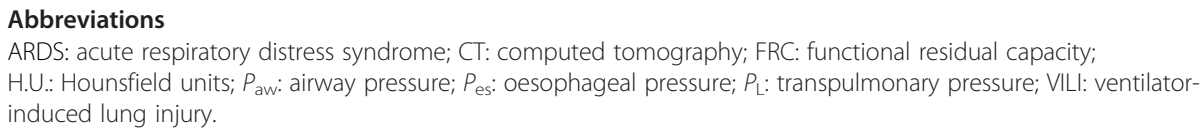

\section{Authors' contributions}

AS conceived, designed and coordinated the study, participated in the acquisition of the data, performed the statistical analysis and drafted the manuscript. AP participated in the design and coordination of the study and helped draft the manuscript. TL conceived the study, participated in its design, participated in the acquisition of the data and helped draft the manuscript. BC, MM and CCS performed the experiments and analysed the lung CT scans. DD performed the surgical preparation and participated in the acquisition of the data. LG coordinated the study and revised the manuscript. All authors read and approved the final version of the manuscript.

\section{Acknowledgements}

We are indebted to Peter Herrmann and Michael Quintel (Department of Anesthesiology, University Hospital of Göttingen, Germany) who kindly provided the Maluna software for the CT scan quantitative analysis and to Luciano Lombardi (Dipartimento di Radiologia, Fondazione IRCCS Ca' Granda-Ospedale Maggiore Policlinico, Milan, Italy) for his valuable technical support. Preliminary results were presented during the 2012 American Thoracic Society International Conference in San Francisco, CA

\section{Author details}

${ }^{1}$ Dipartimento di Fisiopatologica Medico-Chirurgica e dei Trapianti, Università degli Studi di Milano, Via Francesco Sforza 35, 20122 Milan, Italy. Dipartimento di Anestesia, Rianimazione ed Emergenza Urgenza, Fondazione IRCCS Ca' Granda-Ospedale Maggiore Policlinico di Milano, Milan, Italy. ${ }^{3}$ Centro di Ricerche Chirurgiche Precliniche, Fondazione IRCCS Ca' Granda-Ospedale Maggiore Policlinico, Università degli Studi di Milano, Milan, Italy.

Received: 15 April 2015 Accepted: 24 May 2015

Published online: 11 June 2015

\section{References}

1. Slutsky AS (2005) Ventilator-induced lung injury: from barotrauma to biotrauma. Respir Care 50:646-659

2. ARDS Definition Task Force (2012) Acute respiratory distress syndrome: the Berlin definition. JAMA. doi:10.1001/jama.2012.5669

3. Pelosi P, Croci M, Ravagnan I et al (1998) The effects of body mass on lung volumes, respiratory mechanics, and gas exchange during general anesthesia. Anesth Analg 87:654-660

4. Sud S, Friedrich JO, Adhikari NKJ et al (2014) Effect of prone positioning during mechanical ventilation on mortality among patients with acute respiratory distress syndrome: a systematic review and meta-analysis. Can Med Assoc J 186:E381-90. doi:10.1503/cmaj.140081

5. Mure M, Domino KB, Lindahl SG et al (2000) Regional ventilation-perfusion distribution is more uniform in the prone position. J Appl Physiol 88:1076-1083

6. Altemeier WA (2004) Effect of posture on regional gas exchange in pigs. J Appl Physiol 97:2104-2111. doi:10.1152/japplphysiol.00072.2004

7. Pelosi P, Croci M, Calappi E et al (1995) The prone positioning during general anesthesia minimally affects respiratory mechanics while improving functional residual capacity and increasing oxygen tension. Anesth Analg 80:955-960 
8. Pelosi P, Caironi P, Taccone P, Brazzi L (2001) Pathophysiology of prone positioning in the healthy lung and in ALI/ARDS. Minerva Anestesiol 67:238-247

9. Gattinoni L, Pelosi P, Vitale G et al (1991) Body position changes redistribute lung computed-tomographic density in patients with acute respiratory failure. Anesthesiology 74:15-23

10. Pelosi P (2002) Sigh in supine and prone position during acute respiratory distress syndrome. Am J Respir Crit Care Med 167:521-527. doi:10.1164/rccm.200203-1980C

11. Valenza F, Guglielmi M, Maffioletti M et al (2005) Prone position delays the progression of ventilator-induced lung injury in rats: does lung strain distribution play a role? Crit Care Med 33:361-367. doi:10.1097/01.CCM.0000150660.45376.7C

12. Nishimura M, Honda O, Tomiyama N et al (2000) Body position does not influence the location of ventilatorinduced lung injury. Intensive Care Med 26:1664-1669. doi:10.1007/s001340000664

13. Broccard A, Shapiro RS, Schmitz LL et al (2000) Prone positioning attenuates and redistributes ventilator-induced lung injury in dogs. Crit Care Med 28:295-303

14. Nakos G, Batistatou A, Galiatsou E et al (2006) Lung and "end organ" injury due to mechanical ventilation in animals: comparison between the prone and supine positions. Crit Care 10:R38. doi:10.1186/cc4840

15. Santana MCE, Garcia CSNB, Xisto DG et al (2009) Prone position prevents regional alveolar hyperinflation and mechanical stress and strain in mild experimental acute lung injury. Respir Physiol Neurobiol 167:181-188. doi:10.1016/j.resp.2009.04.006

16. Protti A, Cressoni M, Santini A et al (2011) Lung stress and strain during mechanical ventilation: any safe threshold? Am J Respir Crit Care Med 183:1354-1362. doi:10.1164/rccm.201010-17570C

17. Gattinoni L, Protti A, Caironi P, Carlesso E (2010) Ventilator-induced lung injury: the anatomical and physiological framework. Crit Care Med 38:S539-S548. doi:10.1097/CCM.0b013e3181f1fcf7

18. Gattinoni L, Pelosi P, Suter PM et al (1998) Acute respiratory distress syndrome caused by pulmonary and extrapulmonary disease. Different syndromes? Am J Respir Crit Care Med 158:3-11

19. Institute for Laboratory Animal Research (2010) Guide for the care and use of laboratory animals, 8th edition. National Academies Press: Washington, D.C.; 1-246.

20. Protti A, Andreis DT, Monti M et al (2013) Lung stress and strain during mechanical ventilation. Crit Care Med 41:1046-1055. doi:10.1097/CCM.0b013e31827417a6

21. Protti A, lapichino GE, Milesi M et al (2014) Validation of computed tomography for measuring lung weight. pp 1-7. doi:10.1186/s40635-014-0031-0

22. Gattinoni L, Pesenti A, Avalli $L$ et al (1987) Pressure-volume curve of total respiratory system in acute respiratory failure. Computed tomographic scan study. Am Rev Respir Dis 136:730-736. doi:10.1164/ajrccm/136.3.730

23. Gattinoni L, Caironi P, Cressoni M et al (2006) Lung recruitment in patients with the acute respiratory distress syndrome. N Engl J Med 354:1775-1786. doi:10.1056/NEJMoa052052

\section{Submit your manuscript to a SpringerOpen ${ }^{\circ}$ journal and benefit from:}

- Convenient online submission

- Rigorous peer review

- Immediate publication on acceptance

- Open access: articles freely available online

- High visibility within the field

- Retaining the copyright to your article 